\title{
A Combinatorial Result About Points and Balls in Euclidean Space
}

\author{
I. Bárány,' J. H. Schmerl, ${ }^{2}$ S. J. Sidney, ${ }^{2}$ and J. Urrutia ${ }^{3}$
}

'Department of Mathematics, University College, London WCIE 6BT, England

${ }^{2}$ Department of Mathematics, University of Connecticut, Storrs, CT 06268, USA

${ }^{3}$ Department of Computer Science, University of Ottawa, Ottawa, Ontario K1N 9B4, Canada

\begin{abstract}
For each $n \geq 1$ there is $c_{n}>0$ such that for any finite set $X \subseteq \mathbb{R}^{n}$ there is $A \subseteq X,|A| \leq \frac{1}{2}(n+3)$, having the following property: if $B \supseteq A$ is an $n$-ball, then $|B \cap X| \geq c_{n}|X|$. This generalizes a theorem of Neumann-Lara and Urrutia which states that $c_{2} \geq \frac{1}{60}$.
\end{abstract}

A theorem of Neumann-Lara and Urutia [3] is generalized from the plane to arbitrary $n$-dimensional Euclidean space $\mathbb{R}^{n}$, solving Problem 2 of [3]. By an $n$-ball we mean a set of the form

$$
\left\{\left(x_{1}, x_{2}, \ldots, x_{n}\right) \in \mathbb{R}^{n}:\left(x_{1}-a_{1}\right)^{2}+\left(x_{2}-a_{2}\right)^{2}+\ldots+\left(x_{n}-a_{n}\right)^{2} \leq r\right\}
$$

where $\left(a_{1}, a_{2}, \ldots, a_{n}\right) \in \mathbb{R}^{n}$ and $r>0$.

Theorem 1. For each $n \geq 1$ there is $c_{n}>0$ such that for any finite set $X \subseteq \mathbb{R}^{n}$ there is $A \subseteq X,|A| \leq\left[\frac{1}{2}(n+3)\right]$, having the following property: if $B \supseteq A$ is an $n$-ball, then $|B \cap X| \geq c_{n}|X|$.

The bound $\left[\frac{1}{2}(n+3)\right]$ in the theorem will be shown in Theorem 6 to be optimal in quite a strong way. For now, let $X$ be any finite set of points on the moment curve $\alpha(t)=\left(t, t^{2}, t^{3}, \ldots, t^{n}\right),|X|=m \geq n+1$. Then $X$ is the set of vertices of a convex polyhedron (known as the cyclic n-polytope with $m$ vertices) and every [n/2]-element subset $A \subseteq X$ is the set of vertices of one of its faces. (See Sections 4.7 and 7.4 of [2].) Clearly then, for each such $A$ there is an $n$-ball $B$ such that $B \cap X=A$.

The following notation will be used. For a set $S, \mathscr{P}_{n}(S)$ is the set of $n$-element subsets of $S$. If $A \subseteq \mathbb{R}^{n}$, then conv $A$ is the convex hull of $A$. 
Lemma 2. Let $Y \in \mathscr{P P}_{n+3}\left(\mathbb{R}^{n}\right)$. Then there is $A \subseteq Y,|A|=\left[\frac{1}{2}(n+3)\right]$, such that for any $n$-ball $B \supseteq A,(Y \backslash A) \cap B \neq \varnothing$.

Proof. There exist disjoint $A_{1}, A_{2} \subseteq Y$ such that $\left|A_{1}\right|=\left|A_{2}\right|=\left[\frac{1}{2}(n+3)\right]$ and conv $A_{1} \cap$ conv $A_{2} \neq \varnothing$. The argument for obtaining $A_{1}$ and $A_{2}$ is essentially in [1] and [4]. Let $Y=\left\{y_{1}, y_{2}, \ldots, y_{n+3}\right\}$, and then let $\bar{Y}=\left\{\bar{y}_{1}, \bar{y}_{2}, \ldots, \bar{y}_{n+3}\right\} \subseteq \mathbb{R}^{2}$ be its Gale transform. (Here we are assuming, without loss of generality, that $\mathbb{R}^{n}$ is the affine span of $Y$.) For some $y_{1} \in Y$ the line $l$ in $\mathbb{B}^{2}$ through $\vec{y}_{1}$ and the origin divides $\mathbb{R}^{2}$ into two open half-planes $P_{1}, P_{2}$ such that $\left|P_{1} \cap \bar{Y}\right|,\left|P_{2} \cap \bar{Y}\right| \leq\left[\frac{1}{2}(n+3)\right]$. Let $C_{1}, C_{2}, Z \subseteq Y$ be such that $\bar{C}_{1}=P_{1} \cap \bar{Y}, \bar{C}_{2}=P_{2} \cap \bar{Y}$, and $\bar{Z}=l \cap \bar{Y}$. By Lemma 1 of [4], conv $\left(C_{1} \cup Z_{1}\right) \cap \operatorname{conv}\left(C_{2} \cup Z_{2}\right) \neq \varnothing$ whenever $Z_{1} \cup Z_{2}=Z$. But this implies conv $C_{1} \cap$ conv $C_{2} \neq \varnothing$. So just let $A_{1}, A_{2} \subseteq Y$ be disjoint sets such that $C_{1} \subseteq A_{1}, C_{2} \subseteq A_{2}$, and $\left|A_{1}\right|=\left|A_{2}\right|=\left[\frac{1}{2}(n+3)\right]$.

We now claim that either $A=A_{1}$ works or $A=A_{2}$ works.

In order to derive a contradiction, let $a \in \operatorname{conv} A_{1} \cap \operatorname{conv} A_{2}$, and let $B_{1}, B_{2}$ be $n$-balls for which $A_{1} \subseteq B_{1}, A_{2} \subseteq B_{2}$, and $B_{1} \cap A_{2}=\varnothing=B_{2} \cap A_{1}$. Clearly, $B_{1} \cap$ $B_{2} \neq \varnothing$ since $a \in B_{1} \cap B_{2}$, and also $B_{1} \backslash B_{2} \neq \varnothing \neq B_{2} \backslash B_{1}$. Therefore, there is a unique hyperplane $h$ such that $h \cap \partial B_{1}=h \cap \partial B_{2}=\partial B_{1} \cap \partial B_{2}$ (where $\partial B_{1}$ denotes the boundary of $B_{i}$ ). Let $H_{1}, H_{2}$ be the closed half-spaces such that $H_{1} \cap H_{2}=h$, $B_{1} \backslash B_{2} \subseteq H_{1}$, and $B_{2} \backslash B_{1} \subseteq H_{2}$. Then $a \in H_{1} \cap H_{2}=h$, so there must be some $b \in$ $A_{1} \cap h$. But then $b \in B_{2}$, which is a contradiction.

A simple counting argument allows us to deduce Theorem 1 from Lemma 2. This is abstracted in the next lemma.

Lemma 3. Let $S$ be a set, $\mathscr{B}$ a collection of subsets of $S$, and $r>m$ positive integers. Suppose that for each $Y \in \mathscr{P}_{r}(S)$ there is $A \in \mathscr{P}_{m}(Y)$ such that whenever $A \subseteq B \in \mathscr{B}$, then $(Y \backslash A) \cap B \neq \varnothing$. Let $c=(m !(r-m-1) !) / r !$. Then for any finite $X \subseteq S$ with $|X| \geq r$ there is $A \in \mathscr{P}_{m}(X)$ such that whenever $A \subseteq B \in \mathscr{B}$, it follows that $|B \cap X|>$ $c|\boldsymbol{X}|$.

Proof. Let $X \in \mathscr{P}_{t}(S)$ where $t \geq r$. There are sets $A \in \mathscr{P}_{m}(X)$ and $\mathscr{R} \subseteq \mathscr{P}_{r}(X)$ such that $|\mathscr{R}| \geq\left(\begin{array}{l}t \\ r\end{array}\right) /\left(\begin{array}{c}t \\ m\end{array}\right)$ and for each $Y \in \mathscr{R}, A$ is as in the hypothesis of the lemma. We claim that this is the desired $A$.

Suppose that $A \subseteq B \in \mathscr{B}$ and $|B \cap X|=k$. The number of sets $Y \in \mathscr{P}_{r}(X)$ for which $A \subseteq Y$ and $(Y \backslash A) \cap B \neq \varnothing$ is clearly at most $(k-m)\left(\begin{array}{l}t-m-1 \\ r-m-1\end{array}\right)$, so this is an upper bound for $|\mathscr{R}|$. Therefore, $(k-m) \geq c(t-m)$, so $k>c t$.

Lemmas 2 and 3 show that $c_{2} \geq \frac{1}{30}$, improving the constant in [3]. Refinements of this proof show that $c_{2}>\frac{1}{20}$.

The proof of Lemma 3 shows that its conclusion is true on the average, in the sense that as $A$ ranges over $\mathscr{P}_{m}(X)$, the average value of $f(A)=$ $\min \{|B \cap X|: A \subseteq B \in \mathscr{B}\}$ is greater than $c|X|$.

Theorem 1 has several generalizations. We mention just one of them. 
Theorem 4. For each $m \geq\left[\frac{1}{2}(n+3)\right]$ there is $c_{n, m}>0$ such that for any finite $X \subseteq \mathbb{R}^{n}$, $|X| \geq m$, there is $A \in \mathscr{P}_{m}(X)$ having the following property: if $B$ is an $n$-ball and $|A \cap B| \geq\left[\frac{1}{2}(n+3)\right]$, then $|B \cap X| \geq c_{n, m}|X|$.

This theorem is a consequence of Lemma 5 below (which is the analogue of Lemma 2) and a version of Lemma 3 whose statement and proof can easily be supplied.

Let $R_{s}(t)$ be the Ramsey number defined as follows: $R_{s}(t)$ is the least $r$ such that whenever $|Y| \geq r$ and $\mathscr{P}_{s}(Y)=P_{1} \cup P_{2}$, then there is $W \in \mathscr{P}_{i}(Y)$ such that either $\mathscr{P}_{s}(W) \subseteq P_{1}$ or $\mathscr{P}_{s}(W) \subseteq P_{2}$.

Lemma 5. Let $m \geq s=\left[\frac{1}{2}(n+3)\right]$, let $t>m / c_{n}$ be an integer $\left(c_{n}\right.$ is from Theorem $1)$, and let $r=R_{s}(t)$. Suppose $Y \in \mathscr{P}_{r}\left(\mathbb{R}^{n}\right)$. Then there is $A \in \mathscr{P}_{m}(Y)$ such that if $B$ is an $n$-ball and $|B \cap A| \geq s$, then $(Y \backslash A) \cap B \neq \varnothing$.

Proof. Let

$$
P=\left\{Z \in \mathscr{P}_{,}(Y) \text { : for each } n \text {-ball } B \supseteq Z,|B \cap Y| \geq c_{n} t\right\}
$$

By Ramsey's theorem there is $W \in \mathscr{P}_{l}(Y)$ such that $\mathscr{P}_{s}(W) \subseteq P$ or $\mathscr{P}_{s}(W) \cap P=\varnothing$. By Theorem $1, \mathscr{P}_{s}(W) \cap P \neq \varnothing$; hence, $\mathscr{P}_{s}(W) \subseteq P$. Any $A \in \mathscr{P}_{m}(W)$ will do, for $|B \cap A| \geq s$ implies $|B \cap Y| \geq c_{n} t>m=|A|$.

The next theorem shows that the bound on the dimension in Theorem 1 (as well as Lemma 2 and Theorem 4 ) is sharp.

Theorem 6. There is an infinite subset $X \subseteq \mathbb{R}^{n}$ such that whenever $A \subseteq X$ and $|A|<\left[\frac{1}{2}(n+3)\right]$, then there is some $n$-ball $B$ for which $A=B \cap X$.

Proof. Clearly we can assume that $n \geq 3$ and $n$ is odd, so let $n=2 k-1$ where $k \geq 2$. Consider the moment curve $\alpha(t)=\left(t, t^{2}, t^{3}, \ldots, t^{n}\right)$. We will obtain $X$ as $\{\alpha(t): 0<t<\varepsilon\}$ for some appropriately small $\varepsilon>0$.

Suppose $\varepsilon>t_{1} \geq t_{2} \geq \cdots \geq t_{k}>0$. We can find parameters $a_{2 n}, a_{2 n-1}$, $a_{2 n-2}, \ldots, a_{1}, a_{0}$ such that

$$
\left(t-a_{1}\right)^{2}+\left(t^{2}-a_{2}\right)^{2}+\ldots+\left(t^{n}-a_{n}\right)^{2}=a_{0}+\left[\left(t-t_{1}\right)\left(t-t_{2}\right) \ldots\left(t-t_{k}\right)\right]^{2} p(t)
$$

where $p(t)=a_{n+1}+a_{n+2} t+a_{n+3} t^{2}+\cdots+a_{2 n} t^{2 k-2}$. These parameters are uniquely determined, and can be found in the order listed, $a_{i}$ being determined by the coefficients of $t^{j}$ in the above equation. It is easy to see that:

$$
\begin{array}{ll}
a_{j}=1+O(\varepsilon) & \text { if } j \geq 2 k \text { and } j \text { is even; } \\
a_{j}=0+O(\varepsilon) & \text { if } j \text { is odd; } \\
a_{j}=\frac{1}{2}+O(\varepsilon) & \text { if } \quad 1 \leq j \leq 2 k-1 \text { and } j \text { is even; }
\end{array}
$$


and, finally,

$$
a_{0}=\frac{k-1}{4}+O(\varepsilon)
$$

Now just pick $\varepsilon>0$ small enough so that $a_{0}>0$ and $p(t)>0$ whenever $0<t<\varepsilon$. Then if $\varepsilon>t_{1} \geq t_{2} \geq \cdots \geq t_{k}>0$, let $B$ be the $n$-ball with center $\left(a_{1}, a_{2}, \cdots, a_{n}\right)$ and radius $\sqrt{a_{0}}$. Clearly, $X \cap B=\left\{\alpha\left(t_{1}\right), \alpha\left(t_{2}\right), \ldots, \alpha\left(t_{k}\right)\right\}$.

\section{References}

1. J. Eckhoff, Primitive Radon partitions, Mathematika 21 (1974), 32-37.

2. B. Grünbaum, Convex Polytopes, Wiley-Interscience, London, New York, Sydney, 1967.

3. V. Neumann-Lara and J. Urrutia, A combinatorial result on points and circles in the plane, Technical Report TR-85-15, University of Ottawa, 1985.

4. G. C. Shephard, Neighbourliness and Radon's theorem, Mathematika 16 (1969), 273-275.

Received December 18, 1986, and in revised form April 23, 1987.

Note added in proof. Ryan Hayward has shown that $C_{2} \geq \frac{5}{84}$. 